\title{
Reduced activation in ventral striatum and ventral tegmental area during probabilistic decision-making in schizophrenia
}

\author{
Franziska Rausch a,1, Daniela Mier ${ }^{\mathrm{b}, 1}$, Sarah Eifler ${ }^{\mathrm{a}}$, Christine Esslinger ${ }^{\mathrm{c}}$, Claudia Schilling a \\ Frederike Schirmbeck ${ }^{\mathrm{d}}$, Susanne Englisch ${ }^{\mathrm{a}}$, Andreas Meyer-Lindenberg ${ }^{\mathrm{a}}$, Peter Kirsch ${ }^{\mathrm{b}}{ }$, Mathias Zink ${ }^{\mathrm{a}, *}$ \\ a Department of Psychiatry and Psychotherapy, Central Institute of Mental Health, Medical Faculty Mannheim, University of Heidelberg, Germany \\ ${ }^{\mathrm{b}}$ Department of Clinical Psychology, Central Institute of Mental Health, Medical Faculty Mannheim, University of Heidelberg, Germany \\ ${ }^{c}$ MVZ Neurology, Magdeburg, Germany \\ d Academic Medical Centre, Amsterdam University, Netherlands
}

Keywords:

Dopamine

Functional magnetic resonance imaging

Metacognition

Probabilistic reasoning

Schizophrenia

Ventral striatum

\begin{abstract}
A B S T R A C T
Patients with schizophrenia suffer from deficits in monitoring and controlling their own thoughts. Within these so-called metacognitive impairments, alterations in probabilistic reasoning might be one cognitive phenomenon disposing to delusions. However, so far little is known about alterations in associated brain functionality.

A previously established task for functional magnetic resonance imaging (fMRI), which requires a probabilistic decision after a variable amount of stimuli, was applied to 23 schizophrenia patients and 28 healthy controls matched for age, gender and educational levels. We compared activation patterns during decision-making under conditions of certainty versus uncertainty and evaluated the process of final decision-making in ventral striatum (VS) and ventral tegmental area (VTA).

We replicated a pre-described extended cortical activation pattern during probabilistic reasoning. During final decision-making, activations in several fronto- and parietocortical areas, as well as in VS and VTA became apparent. In both of these regions schizophrenia patients showed a significantly reduced activation.

These results further define the network underlying probabilistic decision-making. The observed hypo-activation in regions commonly associated with dopaminergic neurotransmission fits into current concepts of disrupted prediction error signaling in schizophrenia and suggests functional links to reward anticipation. Forthcoming studies with patients at risk for psychosis and drug-naive first episode patients are necessary to elucidate the development of these findings over time and the interplay with associated clinical symptoms.
\end{abstract}

\section{Introduction}

Schizophrenia patients are impaired in detecting, monitoring and controlling their own cognition ("thinking about one's thinking") and synthesizing their mental states. These so-called metacognitive deficits include a reduced ability to appraise and weigh information effectively, to select appropriate responses including decisions based on perceptions and to cope with cognitive limitations (Lysaker et al., 2011, 2013b). Deficits in metacognitive domains are a highly stable property of psychotic patients (Vohs et al., 2014), predicting learning abilities (Tas et al., 2012) and treatment response (So et al., 2014), impairing quality of life (Tas et al., 2013) and outcome (Lysaker et al., 2013a),

\footnotetext{
* Corresponding author at: Central Institute of Mental Health, Department of Psychiatry and Psychotherapy, Medical Faculty Mannheim/ Heidelberg University, P.O. Box 1221 20, D-68072 Mannheim, Germany. Tel.: +49 6211703 2911; fax: + 4962117032525.

E-mail address: mathias.zink@zi-mannheim.de (M. Zink).

${ }^{1}$ Both authors contributed equally.
}

and consequently have been implicated into theories about the formation of delusions (Hemsley and Garety, 1986; Bentall et al., 2009; Speechley et al., 2010; Murray, 2011; So et al., 2012). Therefore specific training interventions targeting metacognitive deficits in psychosis have been invented (Moritz and Woodward, 2007; Van Donkersgoed et al., 2014). However, neural representations of these metacognitive deficits in schizophrenia are widely unclear. The present study was targeted on investigating two key functions of metacognition: probabilistic reasoning and decision-making.

First insight into general neural processes during probabilistic reasoning and decision-making was based on lesion (Xi et al., 2011; Lunt et al., 2012) as well as functional magnetic resonance imaging (fMRI) studies. These studies localized the neural organization of uncertainty (risk or ambiguity) in the course of decision-making represented in a fronto-striatal-thalamic network (Grinband et al., 2006; Bach and Dolan, 2012). Probabilistic reasoning leading to decisions might well be perceived as a specific situation of building up beliefs or perceptions (Deco et al., 2013). Experiences derived from sensory input have to be integrated into prior knowledge. Within this process, prediction errors 
are counterbalanced in a hierarchical Bayesian inference framework between lower- and higher-level systems. On a functional level, the encoding of the precision or uncertainty of prediction errors might be parallel to the encoding of reward uncertainty (Juckel et al., 2006b; Murray et al., 2008; Schultz, 2013). Interestingly, in schizophrenia patients a disruption in prediction error signaling in parallel to a hypo-activation of the ventral striatum (VS) can be found (Lee and Mumford, 2003; Fletcher and Frith, 2009; Friston, 2010; Dura-Bernal et al., 2012). Moreover, it is known from sensitive probabilistic learning or reward prediction tasks (Weickert et al., 2009; Koch et al., 2011; Morris et al., 2012) that schizophrenia patients show differential activation patterns in the fronto-striatal-thalamic network. Hence, these alterations can be assumed to be a neural correlate of deficits in probabilistic reasoning and decision-making in schizophrenia.

Several studies applied the classical beads task regarding probabilistic reasoning and decision-making in healthy volunteers. Blackwood et al. (2004) reported the involvement of cerebellum as well as parietal and occipital cortex. Furl and Averbeck (2011) modified the beads task towards reward-related decision-making and observed less draws until decision than predicted by a Bayesian model. Moreover, in an eventrelated analysis, an increased activation was found in a network comprising parietal, insular, anterior cingulate and striatal regions at the time of decision in comparison to the time of preceding draws. Finally, our group developed a modified version of the beads task and observed activations in cerebellum and prefronto-parietal executive functioning network as well as in medial parieto-occipital regions during the whole process of probabilistic reasoning in healthy volunteers. During the decision process itself, activity in ventral tegmental area (VTA) and VS, comprising the nucleus accumbens (Nacc), was detected (Esslinger et al., 2013).

However, to our knowledge until now there is only one published study exploring decision-making under uncertainty in schizophrenia (Krug et al., 2014). The authors found reduced activation in the prefrontal cortex, but not in subcortical dopaminergic regions in schizophrenia.

In the present study we applied our modified beads task to schizophrenia patients and matched healthy controls. We intended to replicate the activation patterns of our previous study and to evaluate differential activation patterns in schizophrenia patients. It was assumed that patients inappropriately weight evidence during probabilistic reasoning (Fine et al., 2007; Speechley et al., 2010), going along with reduced activation in VS and VTA during final decisionmaking.

\section{Methods}

\subsection{Participants}

This study was approved by the local ethics committee of the Medical Faculty Mannheim of the University of Heidelberg (AZ 2009$296 \mathrm{~N}-\mathrm{MA}$ ). Inpatients were recruited in a stable phase of treatment and fulfilled predefined inclusion criteria: diagnosis of schizophrenia according to the Diagnostic and Statistical Manual, IVth revised edition (DSM-IV R), antipsychotic monotherapy, age between 18 and 60 years, ability to provide informed consent and sufficient German language skills. We excluded patients with severely exacerbated schizophrenia (Positive and Negative Syndrome Scale (PANSS) score $\geq 90$ ), current substance dependence excluding nicotine or other disorders of the central nervous system requiring treatment. Current antipsychotic treatment with second generation antipsychotics was quantified using chlorpromazine (CPZ) equivalents (Andreasen et al., 2010 ), with a mean CPZ equivalent of $406.01 \pm 186$, indicating intermediate dose ranges. Due to anxiety or agitation seven patients were additionally treated with lorazepam. Control subjects were matched for sex, age and levels of education (Table 1 ), had no positive family history of schizophrenia, bipolar disorder or suicide in first-degree relatives and no previous or current psychiatric disorders according to the M.I.N.I. (Mini-International Neuropsychiatric Interview) or psychopharmacological therapy.

\subsection{Psychometric rating scales and neuropsychological characterization}

Psychotic symptoms were characterized by trained raters (FR, SE, SE) using PANSS and PSYRATS (Psychotic Symptoms Rating Scale). We further evaluated negative symptoms (Scale for the Assessment of Negative Symptoms: SANS), comorbid depressive symptoms (Calgary Depression Scale for Schizophrenia: CDSS), general severity of illness (Clinical Global Impression: CGI) and psychosocial functioning (Global Assessment of Functioning: GAF; Personal and Social Performance Scale: PSP).

Neuropsychological assessment included the Trail Making Tests A and B (TMT-A, -B), the Wisconsin Card Sorting Test (WCST) and the Multiple Choice Word Test version B (MWT-B, Table 1).

\subsection{Modified beads task}

Our modification of the classical beads task had been described earlier (Esslinger et al., 2013). In short, subjects viewed fish of two colors jumping out of a lake and had to decide from which of two lakes they were coming at a color ratio of $80 / 20 \%$ or $20 / 80 \%$. After each fish, subjects were asked if they wanted to see another fish and could answer the question by pressing according buttons. The colored fish were presented in a previously defined fashion (e.g. 1-1-1-2-1-1-1-1-2-1), recapitulated eight times with alternative starting points. For methodological reasons, the number of fish per block was restricted to ten. After presentation of the selected number of fish or a maximum of ten fish, subjects had to decide for one lake and to rate on a four-point scale how confident they were regarding their decision $(1=$ a little uncertain, $2=$ fairly certain, $3=$ very certain, $4=$ totally certain). In the control condition, subjects had to indicate the colors of fish. To ensure a standardized duration of the experiment, unequal lengths of the experimental blocks were counterbalanced by the number of control trials. Eight experimental blocks and eight control blocks were presented adding up to eight times at $2.04 \mathrm{~min}$ (16.32 $\mathrm{min}$ for the whole experiment).

\subsection{Acquisition and evaluation of fMRI data}

Blood oxygen level-dependent (BOLD) fMRI was performed on a $3 \mathrm{~T}$ Siemens Trio (Siemens Medical Systems, Erlangen, Germany) by using echo-planar imaging (28 axial slices; 4-mm thickness; 1-mm gap; TR/ TE 2000/28 ms; FOV $19.2 \mathrm{~cm}$; matrix $64 \times 64$ ). FMRI data was analyzed using SPM8 (www.fil.ion.ucl.ac.uk/spm/software/spm8/) as described earlier (Esslinger et al., 2013). Prior to analysis, data was preprocessed including realignment, slice timing, and normalization to a standard EPI template volume with resampling to a $3 \times 3 \times 3 \mathrm{~mm}$ voxel size and smoothing with a $9 \mathrm{~mm}$ full-width half-maximum Gaussian filter. Task-specific brain activation was analyzed in a hybrid model according to Visscher et al. (2003). We compared neural processes during lake reasoning (decision out of which lake fish were jumping) versus color naming (decision about the color of fish). In addition, we compared the neural response to the last fish, followed by a final decision, in comparison to all previous fish that were not followed by a decision for a lake. To control for possible movement-related artifacts, six further regressors were entered into the model, containing information from the realignment. Contrasts of interest were entered into second-level random-effects group analyses, applying one-sample and two-sample T-tests. Since groups slightly differed in gender distribution, gender was used as a covariate. 
Table 1

Sociodemographic, psychopathological and neurocognitive characteristics of study samples: Data is reported as mean \pm standard deviation (SD). Abbreviations: CDSS: Calgary Depression Scale for Schizophrenia, CGI-S: Clinical Global Impression - Severity subscore, CPZ: Chlorpromazine equivalent, f: female, GAF: Global Assessment of Functioning, m: male, mg: milligram, MWT-B: Multiple Choice Word Test (version B), n.a.: not applicable, n.s.: not significant, PANSS: Positive and Negative Syndrome Scale, PSP: Personal and Social Performance Scale, PSYRATS: Psychotic Symptoms Rating Scale, sec: seconds, TMT: Trail Making Test; WCST: Wisconsin Card Sorting Test.

\begin{tabular}{|c|c|c|c|}
\hline & Patients $(\mathrm{n}=23$ ) & Controls $(\mathrm{n}=28)$ & Comparison \\
\hline \multicolumn{4}{|l|}{ Sociodemographics } \\
\hline Age & $33.17 \pm 9.114$ & $35.79 \pm 12.075$ & n.s. \\
\hline Gender & $f=7, m=16$ & $\mathrm{f}=13, \mathrm{~m}=15$ & $\begin{array}{l}\mathrm{X}^{2} \text {-test: } \mathrm{p}=0.244, \mathrm{n} . \mathrm{s} . \\
\text { Fisher-test: } \mathrm{p}=0.191 \text {, n.s. }\end{array}$ \\
\hline Duration of education (years) & $15.20 \pm 3.278$ & $15.41 \pm 3.073$ & n.s. \\
\hline Time spent in school (years) & $10.96 \pm 1.745$ & $11.25 \pm 1.555$ & n.s. \\
\hline \multicolumn{4}{|l|}{ Clinical properties } \\
\hline Duration of illness (years) & $7.48 \pm 6.802$ & n.a. & n.a. \\
\hline CPZ-equivalents (mg) & $406.01 \pm 185.994$ & n.a. & n.a. \\
\hline \multicolumn{4}{|l|}{ PANSS } \\
\hline Total Score & $63.57 \pm 13.107$ & n.a. & n.a. \\
\hline Positive symptoms & $13.70 \pm 4.258$ & n.a. & n.a. \\
\hline Negative symptoms & $16.91 \pm 4.776$ & n.a. & n.a. \\
\hline Global psychopathology & $32.96 \pm 7.547$ & n.a. & n.a. \\
\hline \multicolumn{4}{|l|}{ Additional psychometric Scales } \\
\hline SANS & $24.60 \pm 24.632$ & n.a. & n.a. \\
\hline CDSS & $3.00 \pm 2.981$ & n.a. & n.a. \\
\hline PSP & $52.80 \pm 13.903$ & n.a. & n.a. \\
\hline GAF & $44.10 \pm 10.867$ & n.a. & n.a. \\
\hline CGI-Severity & $3.90 \pm 1.101$ & n.a. & n.a. \\
\hline \multicolumn{4}{|l|}{ PSYRATS } \\
\hline Amount of preoccupation & $1.20 \pm 1.398$ & n.a. & n.a. \\
\hline Duration of preoccupation & $1.40 \pm 1.430$ & n.a. & n.a. \\
\hline Conviction & $1.70 \pm 1.636$ & n.a. & n.a. \\
\hline Amount of distress & $1.60 \pm 1.647$ & n.a. & n.a. \\
\hline Intensity of distress & $1.80 \pm 1.620$ & n.a. & n.a. \\
\hline Disruption & $1.30 \pm 1.337$ & n.a. & n.a. \\
\hline \multicolumn{4}{|l|}{ Processing speed (TMT) } \\
\hline TMT-A (sec) & $38.74 \pm 13.515$ & $24.61 \pm 6.762$ & T-test: $\mathrm{p}<0.001$ \\
\hline TMT-B (sec) & $83.22 \pm 30.179$ & $57.50 \pm 19.207$ & T-test: $\mathrm{p}=0.003$ \\
\hline \multicolumn{4}{|l|}{ Executive functioning (WCST) } \\
\hline Categories completed & $6.44 \pm 1.464$ & $6.68 \pm 1.156$ & n.s. \\
\hline Total Trials & $89.06 \pm 15.821$ & $77.36 \pm 8.010$ & T-test: $\mathrm{p}=0.008$ \\
\hline Total Errors (\%) & $25.69 \pm 15.099$ & $17.36 \pm 4.499$ & T-test: $\mathrm{p}=0.035$ \\
\hline Perseveration Score (\%) & $24.04 \pm 14.429$ & $12.10 \pm 8.563$ & T-test: $\mathrm{p}=0.001$ \\
\hline Concept perseverations & $1.78 \pm 2.487$ & $0.18 \pm 0.390$ & T-test: $\mathrm{p}=0.015$ \\
\hline Failure to maintain set & $1.33 \pm 1.970$ & $1.00 \pm 1.846$ & n.s. \\
\hline \multirow[t]{2}{*}{ Multiple choice word test version B (MWT-B) } & $25,409 \pm 5198$ & $28,571 \pm 4857$ & T-test: $\mathrm{p}=0.031$ \\
\hline & $\mathrm{N}=22$ & $\mathrm{~N}=28$ & \\
\hline Estimated verbal IQ & $99,500 \pm 13,821$ & $107,929 \pm 15,592$ & T-test: $\mathrm{p}=0.052$ \\
\hline
\end{tabular}

\subsection{Statistics}

Socio-demographic, psychometric and behavioral parameters were analyzed using the Statistical Package for Social Sciences (IBM SPSS version 20.0, Chicago, IL, US). We tested for group-specific differences of means and applied student's T-tests, Fisher's exact test and $\mathrm{X}^{2}$-test. Correlations between psychopathological characteristics and neurocognitive abilities were expressed by Pearson's correlation coefficient after correction for multiple testing. Regarding statistical inference of fMRI data, a threshold of $\mathrm{p}<0.05$ with family wise error (FWE) correction for multiple testing was applied and a minimal cluster size threshold of $k=5$ adjacent voxels was set. To specifically study activation in VS and VTA, region of interest (ROI) analyses were performed. Statistical threshold for ROI analyses was $\mathrm{p}<0.05$, small volume (sv) corrected. As described earlier (Esslinger et al., 2013), masks for VTA and VS, comprising left and right Nacc, were created with MARINA (masks for region of interest analyses) (Walter et al., 2003), according to an anatomical atlas (Duvernoy, 1995).

\section{Results}

Groups of 23 patients and 28 controls, matched for age, gender and education were included into final data analysis. Before, five patients and one control subject had to be excluded (lack of understanding the task: $\mathrm{n}=3$, gross brain abnormalities: $\mathrm{n}=1$, strong movements: $\mathrm{n}=2$ ). Patients were characterized as moderately ill and showed marked impairment in neurocognitive domains (Table 1).

\subsection{Modified beads task}

The number of stimuli needed for decision (draws to decision: DTD) was averaged over the eight repeats of the task. The mean $\mathrm{DTD}_{8}$ over all blocks differed between patients $(4.97 \pm 1.4)$ and controls $(4.06 \pm$ $1.5, \mathrm{~T}=-2,18, \mathrm{df}=49, \mathrm{p}=0.034$ ) with a mean level of certainty of $2.76 \pm 0.8$ in patients and $3.06 \pm 0.7$ in controls (definition: fairly certain $=2$, very certain $=3, \mathrm{~T}=1,44, \mathrm{df}=49, \mathrm{p}=0.155)$. $\mathrm{DTD}_{8} \mathrm{~s}$ and levels of certainty did not change significantly within groups during the eight blocks, nor between groups.

\section{2. fMRI}

\subsubsection{General activation patterns}

Decision-making during lake reasoning versus color naming was found to be associated with increased activation in several frontal and parietal regions, inferior temporal gyrus and globus pallidus (Table 2 and Fig. 1). ROI analyses revealed significant effects in VTA (coordinates: $9-19-14, \mathrm{~T}=4.53, \mathrm{p}=0.001$ sv-corrected, $\mathrm{k}=126$ ), right VS (coordinates: $12111, \mathrm{~T}=4.55, \mathrm{p}=0.001$ sv-corrected, $\mathrm{k}=67$ ), and on a trend level in left VS (coordinates: $-2117-8, \mathrm{~T}=2.84, \mathrm{p}=$ 0.062 sv-corrected, $\mathrm{k}=20$ ). 
Table 2

Activation during probabilistic reasoning versus control condition, $\mathrm{p}<0.05$, FWE corrected for the whole brain. $\mathrm{BA}=$ Brodmann area, $\mathrm{Tmax}=$ maximal T-value in the cluster, coordinates $=$ MNI (Montreal Neurological Institute) coordinates of the peak voxel in the cluster. $\mathrm{k}=$ cluster-size, sub-cluster peaks are inserted.

\begin{tabular}{|c|c|c|c|c|c|c|}
\hline \multirow{2}{*}{$\begin{array}{l}\text { Activation } \\
\text { Area }\end{array}$} & \multirow[b]{2}{*}{$\mathrm{BA}$} & \multirow[b]{2}{*}{ Cluster } & \multicolumn{3}{|l|}{ MNI } & \multirow[b]{2}{*}{ Tmax } \\
\hline & & & $\mathrm{x}$ & $\mathrm{y}$ & $\mathrm{z}$ & \\
\hline Inferior parietal lobule & 40 & 6836 & 51 & -43 & 46 & 13.10 \\
\hline Superior parietal lobule & 7 & & 30 & -70 & 49 & 12.35 \\
\hline Precuneus & 19 & & 30 & -76 & 31 & 11.25 \\
\hline Inferior prefrontal gyrus & 47 & 2200 & 36 & 20 & -2 & 11.80 \\
\hline Middle frontal gyrus & 10 & & 39 & 53 & -5 & 11.27 \\
\hline Inferior prefrontal gyrus & 47 & & 30 & 26 & -11 & 10.69 \\
\hline Medial frontal gyrus & 8 & 471 & 3 & 29 & 43 & 9.90 \\
\hline Inferior prefrontal gyrus & 47 & 115 & -30 & 20 & -5 & 8.77 \\
\hline Inferior prefrontal gyrus & 47 & 267 & -42 & 44 & -5 & 8.62 \\
\hline Superior frontal gyrus & 11 & & -21 & 47 & -14 & 6.80 \\
\hline Middle frontal gyrus & 46 & 519 & -45 & 26 & 31 & 8.03 \\
\hline Middle frontal gyrus & 9 & & -45 & 8 & 34 & 7.45 \\
\hline Middle frontal gyrus & 6 & & -42 & 2 & 58 & 6.14 \\
\hline Inferior temporal gyrus & 20 & 13 & 63 & -28 & -20 & 6.26 \\
\hline Pallidus & & 12 & -12 & -4 & -5 & 6.17 \\
\hline Middle frontal gyrus & 6 & 13 & -18 & 17 & 61 & 5.88 \\
\hline
\end{tabular}

Comparing the activation during the last fish with a decision with activation related to all previous fish, we observed increased activation in several frontal and parietal areas, in putamen, cerebellum and midbrain. ROI analyses showed a significant effect in VTA (coordinates: $-6-16-8, \mathrm{~T}=5.79, \mathrm{p}<0.001$ sv-corrected, $\mathrm{k}=124$ ) and VS bilaterally (left coordinates: $-215-2, \mathrm{~T}=5.14, \mathrm{p}<0.001$ sv-corrected $\mathrm{k}=70$; right coordinates: $1811-2, \mathrm{~T}=5.45, \mathrm{p}<0.001$ sv-corrected, $\mathrm{k}=91$ ) (Table 3 and Fig. 2 ).

\subsubsection{Between group comparisons}

Whole brain analyses revealed no significant differences between groups, given the strict significance threshold. However, ROI analyses
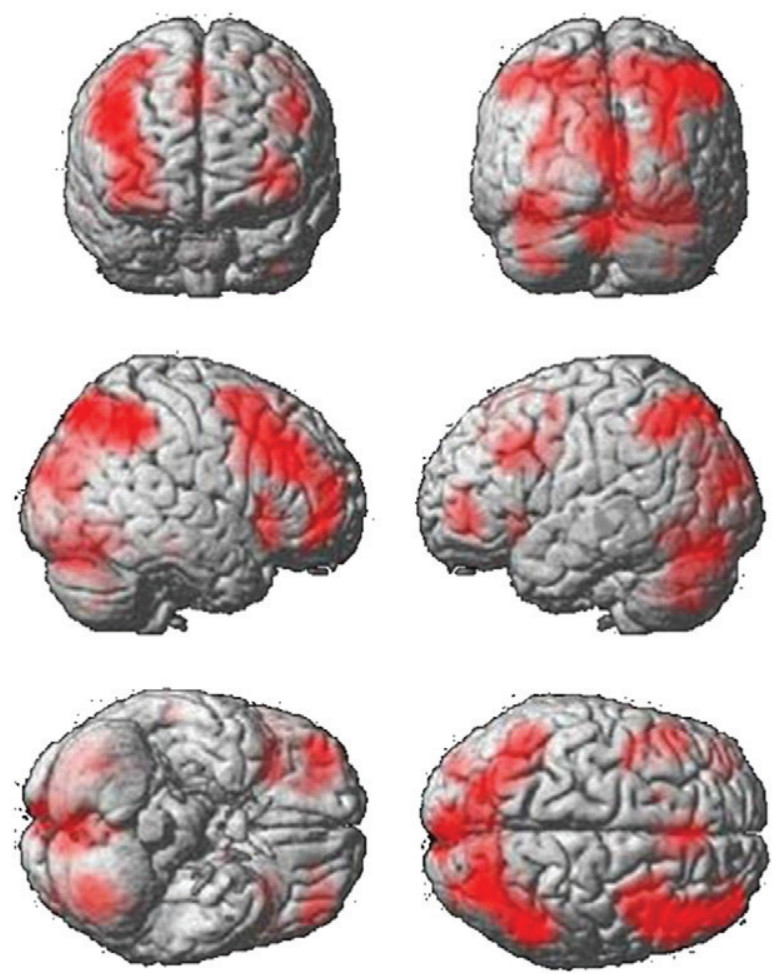

Fig. 1. Brain activation during the modified beads task. Across all presented fish, the experimental condition (lake reasoning) is compared with the control condition (color naming). Level of significance: $\mathrm{p}<0.05 \mathrm{FWE}-$ corrected for the whole brain.
Table 3

Activation during presentation of the last versus all preceding fish, $\mathrm{p}<0.05 \mathrm{FWE}-$ corrected for the whole brain. $\mathrm{BA}=$ Brodmann area, Tmax $=$ maximal T-value in the cluster, coordinates $=$ MNI (Montreal Neurological Institute) coordinates of the peak voxel in the cluster. $\mathrm{k}=$ cluster-size, sub-cluster peaks are inserted.

\begin{tabular}{|c|c|c|c|c|c|c|}
\hline \multirow{2}{*}{$\frac{\text { Activation }}{\text { Area }}$} & \multirow[b]{2}{*}{ BA } & \multirow[b]{2}{*}{ Cluster } & \multicolumn{3}{|l|}{ MNI } & \multirow[b]{2}{*}{ T-value } \\
\hline & & & $\mathrm{x}$ & $\mathrm{y}$ & z & \\
\hline Inferior prefrontal gyrus & 47 & 133 & 33 & 20 & -2 & 8.37 \\
\hline Medial frontal gyrus & 6 & 275 & -9 & 5 & 55 & 7.07 \\
\hline Dorsal anterior cingulate cortex & 32 & & 9 & 8 & 49 & 6.46 \\
\hline Middle frontal gyrus & 6 & & -27 & -10 & 52 & 6.28 \\
\hline Putamen & & 73 & -15 & 5 & 10 & 5.97 \\
\hline Cerebellum & & 39 & 0 & -43 & -38 & 5.91 \\
\hline Midbrain & & 37 & -6 & -16 & -8 & 5.79 \\
\hline Midbrain & & & 6 & -25 & -5 & 5.68 \\
\hline Midbrain & & & -3 & -28 & -5 & 5.29 \\
\hline Inferior parietal lobule & 40 & 17 & -39 & -34 & 49 & 5.56 \\
\hline Lingual gyrus & 18 & 11 & 24 & -76 & -5 & 5.54 \\
\hline putamen & & 27 & 18 & 11 & -2 & 5.45 \\
\hline Putamen & & & 21 & 5 & 7 & 5.44 \\
\hline Precentral gyrus & 6 & 39 & 36 & -10 & 61 & 5.42 \\
\hline Middle frontal gyrus & 6 & & 30 & -7 & 52 & 5.32 \\
\hline Middle frontal gyrus & 6 & & 39 & -7 & 52 & 5.22 \\
\hline Claustrum & & 10 & -30 & 20 & 1 & 5.34 \\
\hline Precentral gyrus & 6 & 6 & -54 & 2 & 37 & 5.22 \\
\hline
\end{tabular}

revealed significantly reduced activation in schizophrenia patients compared to healthy controls in VTA (coordinates: $3-13-6, \mathrm{~T}=$ 3.74, $\mathrm{p}=0.007 \mathrm{sv}$-corrected, $\mathrm{k}=83$ ) and right VS (coordinates: $215-5, \mathrm{~T}=2.93 ; \mathrm{p}=0.038$ sv-corrected, $\mathrm{k}=22$ ) (Fig. 3) for final decision-making (last fish vs. all previous fish). Moreover, ROIanalyses were repeated without gender as covariate, as well as with IQ as covariate. In both cases, differences between groups in VTA and VS remained stable. Furthermore, ROI-analyses for fusiform gyrus and hippocampus (defined and created with the WFU_pickatlas) were performed to investigate a possible general hypo-activity in the schizophrenia sample. However, no significant differences between groups were found in these regions.

While VTA- and VS-activations were significantly lower in patients than in controls, the amount of BOLD response in both VS and VTA did not correlate with the mean number of fish, neither in controls nor in patients. In patients, the anti-dopaminergic intensity of antipsychotic treatment (CPZ-equivalents) was neither correlated with the mean number of fish, nor with VTA- or VS-activation. A comparison of patients with or without concomitant benzodiazepine treatment did not reveal activation differences. Severity of psychotic symptoms did neither correlate with mean number of fish, nor with certainty at the time point of decision or brain activation.

\subsubsection{Correlations between fMRI activation and behavior and cognition}

Control subjects, but not schizophrenia patients, showed a significant correlation between WCST item "failure to maintain set" and mean number of fish $(r=0.517, p=0.005)$, which remained significant even after correction for multiple testing. In the total sample, we further observed a significant negative correlation between VTAactivation and WCST "total errors" $(\mathrm{r}=-0.302, \mathrm{p}=0.041)$ as well as TMT-A reaction times $(\mathrm{r}=-0.334, \mathrm{p}=0.017)$.

\section{Discussion}

The present study was set up for investigating neuronal activation in schizophrenia during probabilistic reasoning. As hypothesized, patients showed significantly reduced activation in VS and VTA during final decision-making. The findings fit into current concepts of disrupted prediction error signaling in schizophrenia and suggest functional links to dopaminergic dysfunctions and reward anticipation.

Several previous fMRI studies addressed decision-making and errorrelated learning in schizophrenia and suggested functional alterations 

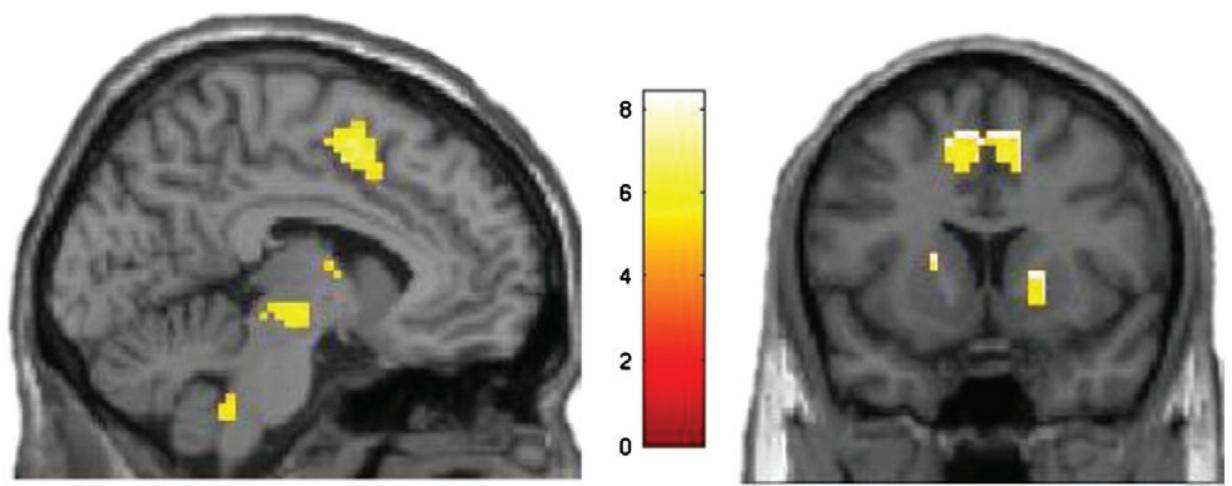

Fig. 2. Brain activation related to the event of decision-making. Activation in response to the last fish with a decision in comparison to all previous fish. Level of significance: $\mathrm{p}<0.05 \mathrm{FWE}-$ corrected for the whole brain.

in VS. Morris et al. (2012) found abnormal differential activation of VS in response to expected versus unexpected outcomes. Waltz et al. (2007)) and Gold et al. (2012) administered probabilistic selection tasks to schizophrenia patients and observed deficits in reinforcement learning as a hint towards striatal-cortical dysfunction. Furthermore, Koch et al. (2011) reported decreased activation in a fronto-striatocingulate network in schizophrenia. In the study of Weickert et al. (2009) schizophrenia patients differentially activated a compensatory neural network, consisting of dorsolateral prefrontal, cingulate, parahippocampal and parietal cortex in the absence of normal frontalstriatal function. Recently, Krug et al. were able to show reduced activation in prefrontal cortex during decision-making under uncertainty (Krug et al., 2014).

Closely related to our approach, three further fMRI studies applied modifications of the classical beads task to healthy volunteers (Blackwood et al., 2004; Furl and Averbeck, 2011; Esslinger et al., 2013). We could largely replicate the activation patterns observed by Blackwood et al. (2004)). Furl and Averbeck (2011) used a rewardrelated version and reported higher activation during decision-making in a network also comprising striatal regions. The proposed functional link to reward anticipation (Juckel et al., 2006a, 2006b; Ziauddeen and Murray, 2010) was further supported by our pilot investigation (Esslinger et al., 2013). In a mixed model analysis, without considering the process of decision immediately, but evolving across several preceding trials, we found activations in VTA and VS (comprising Nacc) during decision-making (Esslinger et al., 2013).

As a next step, we applied the modified beads task to schizophrenia patients and observed reduced activations in the ROIs VTA and right VS in parallel to the cognitive process of decision-making. These findings might be interpreted within theories about decision-making in schizophrenia (Fletcher and Frith, 2009) and the functional representation of reward uncertainty by dopaminergic neurotransmission (Fiorillo et al., 2003; Tobler et al., 2005; Schultz et al., 2008; Schultz, 2013). It was shown that schizophrenia patients differ from controls in their ability

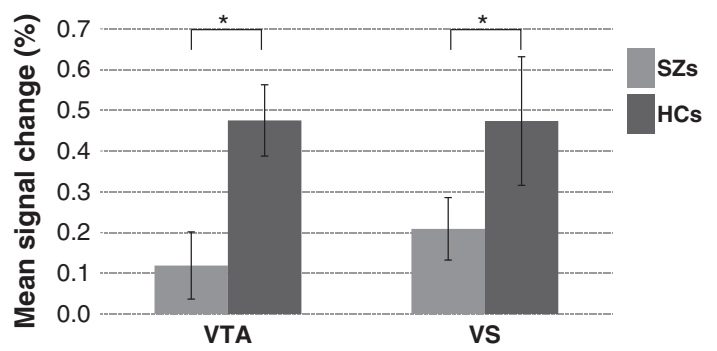

Fig. 3. Hypo-activation during final decision-making in VTA and VS (comprising the Nacc) in the patient group (both, $\mathrm{p}<0.05 \mathrm{sv}$-corrected). Mean signal change is shown for the contrast last fish $>$ all previous fish, separately for both groups. Level of significance (for display purposes): $\mathrm{p}<0.005$, (uncorrected). to propagate prediction errors in a hierarchical Bayesian inference framework between lower- and higher-level systems (Lee and Mumford, 2003; Fletcher and Frith, 2009; Friston, 2010; Dura-Bernal et al., 2012). The supposed disruption in prediction error signaling has been attributed to hypo-activity in VS, because dopamine signaling is thought to encode the precision or uncertainty of prediction errors (Juckel et al., 2006b; Murray et al., 2008). Prediction error signaling and data gathering for decisions represent different aspects of the Bayesian inference process, while information might well be apprehended as a form of reward. As a function of reward probability, the expected reward increases linearly, while the risk or uncertainty of reward follows an inverted U-shaped curve with a theoretical maximum at a reward probability of $50 \%$ (Schultz et al., 2008; Schultz, 2013). Patients seem to differ in how they weight evidence in the beads task (Fine et al., 2007; Moritz et al., 2012) and in a "faulty appraisal" they might underestimate the uncertainty of their choices early in the decision process and show a lowered threshold for making decisions in an ambiguous context (Averbeck et al., 2011; Rubio et al., 2011; Veckenstedt et al., 2011). In line with the assumption of a reduction in tonic dopamine availability (Goto et al., 2007), we suggest either a left-shift of the above mentioned inverted U-shaped curve or a global reduction of the absolute values that explains the observed hypo-activation during decision-making in schizophrenia.

Noteworthy, in our modified beads task patients used more stimuli than healthy controls until decision, as averaged over the eight repeats. We applied similar ratios of stimuli as previous investigations with the beads task have used. Nevertheless, the experimental setting during scanning in the fMRI markedly differs from the commonly used test conditions and might have rendered the patients even more cautious in decision-making than controls. Noteworthy, in several other studies schizophrenia patients did not present the canonical jumping to conclusion (JTC) behavior (for review see Ziegler et al., 2012). Further influencing factors on test behavior might have been the subtype of delusions (Garety et al., 2012), the chronic course of illness, and the treatment effects of antipsychotic medication. Importantly, Peters and Garety had observed a normalization of hasty decision-making during remission of delusions (Peters and Garety, 2006) and Woodward et al. reported significant correlations between normalization of decisionmaking and remission of delusions (Woodward et al., 2009b). Since our sample was low on positive symptoms severity, no severe forms of hasty decision-making can be expected. It might be further assumed that patients were aware of their difficulties in decision-making, adopted a more cautious approach due to experienced negative consequences and might have over-compensated their primary metacognitive deficit on the behavioral level. Aside from the behavioral level, the primary endpoint of this study was the differential neural activation pattern during task performance. In similar studies the lack of a behavioral phenotype regarding decision-making under uncertainty also did not preclude the observation of decreased activation in a fronto-striato-cingulate network (Koch et al., 2011) or in prefrontal 
cortex (Krug et al., 2014). In parallel, our study allowed us to reveal the underlying neural processes of decision-making even without observing the JTC-phenotype that had been documented in the majority of schizophrenia studies. These might present an intermediate phenotype of deficient probabilistic reasoning in schizophrenia, independent of factors that modify and compensate the behavioral phenotype.

It has been proposed that neurocognitive capacities, most importantly executive functioning, might modulate probabilistic decisionmaking (Lysaker et al., 2008; Woodward et al., 2009a; Lincoln et al., 2010b). In agreement with an assumed association between cognitive flexibility and decision-making, we observed an inverse correlation between VTA-activation and cognitive flexibility in our entire study sample. However, this correlational finding has to be interpreted with care, due to the group differences in VTA-activation, as well as cognitive flexibility. The counterintuitive positive relationship in control subjects between the mean number of fish drawn to decision $\left(\mathrm{DTD}_{8}\right)$ and the WCST-item "failure to maintain set" has not been obtained in schizophrenia patients. Other pre-described neurocognitive domains such as working memory (Broome et al., 2007; Ormrod et al., 2011), verbal learning and memory or mastery (Lysaker et al., 2011; Buck et al., 2012) have not been evaluated in our study. In addition, while estimated verbal intelligence did not explain altered decision-making in schizophrenia, but its association with observer-rated delusions (Lincoln et al., 2010a), small effects of intelligence levels cannot be excluded. However, using intelligence as covariate did not diminish group differences. Furthermore, future studies should investigate how emotional states influence the ability to think about one's own thinking, as well as perception and decisions (Garety et al., 2012).

\section{Limitations}

Potential confounding effects of chronic course of illness, fMRIspecific testing conditions, medication, the resulting treatment response, as well as learned and counteracting efforts of the patients might have inhibited observing a JTC-bias in our schizophrenia sample. Hence, our results add to the knowledge about neural correlates of probabilistic reasoning and decision-making in schizophrenia, but do not help explaining the neural correlates of the JTC-bias in particular. Notably, we did not find influences of CPZ equivalents neither on a behavioral level, nor regarding BOLD responses. This is in concert with the general tendency in the literature (So et al., 2010) and a recent experimental study, where dopaminergic treatment modulations using haloperidol or L-dopa did not modify the number of draws-todecision or the probability threshold (Andreou et al., 2013). Nevertheless, antipsychotic medication might influence dopaminergic signaling during decision-making, independent of the strength of just dopaminergic binding. In addition, neurochemical signaling of uncertainty of prediction errors might well involve not only dopamine, but also amino acid neurotransmitters, acetylcholine and norepinephrine (Yu and Dayan, 2005). Metacognitive deficits are certainly not restricted to schizophrenia (Dimaggio et al., 2013; Ladegaard et al., 2014), but represent general patho-mechanisms with important implications for other mental disorders, too.

\section{Conclusions}

This fMRI study allows insight into activation patterns of probabilistic reasoning and decision-making in schizophrenia. Compared to behavioral studies, the evaluation of fMRI can be considered as more sensitive, more closely related to the primary cognitive processes and more independent from factors that might confound the behavioral phenotype. Applying this method, we found hypo-activation in VS and VTA during final decision-making in schizophrenia patients. These results suggest a dysregulated dopaminergic functioning during these cognitive processes in schizophrenia. Future studies should focus on longitudinal investigations starting with at risk mental states for psychosis in order to describe the neuronal structures and neurochemical correlates of metacognitive deficits in schizophrenia and improve our understanding of the formation of delusions.

\section{Funding}

M.Z., A.M.-L., and P.K. were funded by the Deutsche Forschungsgesellschaft (DFG, http://www.dfg.de, projects ZI1253/3-1, ZI1253/3-2, KI 576/14-2, ME 1591/6-2). S.E. received funding by the LGFG (Landesgraduiertenförderungsgesetz), C.S. and D.M. by the Olympia-Morata Program, and F.S. by the Evangelisches Studienwerk. The funders had no role in study design, data collection and analysis, decision to publish or preparation of the manuscript.

\section{Contributors}

Franziska Rausch: Recruitment of patients, neurocognitive testing, metacognitive testing, fMRI, analysis of data, discussion and interpretation of data, preparation of the manuscript.

Daniela Mier: fMRI, analysis of data, discussion and interpretation of data, preparation manuscript.

Sarah Eifler: Recruitment of patients, neurocognitive testing, metacognitive testing, fMRI, analysis of data, discussion and interpretation of data, proof-reading and editing of the manuscript.

Christine Esslinger: Setup of the experiment, pilot studies fMRI, discussion and interpretation of data, proof-reading and editing of the manuscript.

Claudia Schilling: Recruitment of patients, neurocognitive testing, fMRI, analysis of data, discussion and interpretation of data, proof-reading and editing of the manuscript. Frederike Schirmbeck: Setup of the experiment, neurocognitive testing, discussion and interpretation of data, proof-reading and editing of the manuscript.

Susanne Englisch: Recruitment of patients, discussion and interpretation of data, proof-reading and editing of the manuscript.

Andreas Meyer-Lindenberg: Institutional support for the project, Co-applicant of the scientific grant, discussion and interpretation of data, proof-reading and editing of the manuscript.

Peter Kirsch: Co-applicant of the scientific grant, fMRI, analysis of data, discussion and interpretation of data, proof-reading and editing of the manuscript.

Mathias Zink: Applicant of the scientific grant, setup of the experiment, recruitment of patients, discussion and interpretation of data, preparation of the manuscript.

\section{Conflicts of interest}

Franziska Rausch, Daniela Mier, Sarah Eifler, Christine Esslinger, Claudia Schilling and Peter Kirsch: NO COI. Frederike Schirmbeck was supported by a grant of Evangelisches Studienwerk, Susanne Englisch has received travel expenses and consultant fees from AstraZeneca, Bristol-Myers Squibb GmbH \& CoKGaA, Eli-Lilly, Janssen Cilag, Otsuka Pharma, Pfizer Pharma and Servier. Andreas Meyer-Lindenberg receives consultant fees and travel expenses from AstraZeneca, Hoffmann-La Roche, Lundbeck Foundation. Speaker's fees from Pfizer Pharma, Lilly Deutschland, Glaxo SmithKline, Janssen Cilag, Bristol-Myers Squibb, Lundbeck, Servier and AstraZeneca. Mathias Zink received unrestricted scientific grants of the European Research Advisory Board (ERAB), German Research Foundation (DFG), Pfizer Pharma GmbH, Servier and Bristol Myers Squibb Pharmaceuticals; further speaker and travel grants were provided from Astra Zeneca, Lilly, Pfizer Pharma GmbH, Bristol Myers Squibb Pharmaceuticals, Otsuka, Servier, Lundbeck and Janssen Cilag.

\section{Acknowledgments}

We are grateful to all participants, to Dagmar Gass for assistant data acquisition and to Prof. Dr. Steffen Moritz (UKE Hamburg, Germany) for helpful discussion.

\section{References}

Andreasen, N.C., Pressler, M., Nopoulos, P., Miller, D., Ho, B.C., 2010. Antipsychotic dose equivalents and dose-years: a standardized method for comparing exposure to different drugs. Biol. Psychiatry 67, 255-262.

Andreou, C., Moritz, S., Veith, K., Veckenstedt, R., Naber, D., 2013. Dopaminergic modulation of probabilistic reasoning and overconfidence in errors: a double-blind study. Schizophr. Bull. http://dx.doi.org/10.1093/schbul/sbt064.

Averbeck, B.B., Evans, S., Chouhan, V., Bristow, E., Shergill, S.S., 2011. Probabilistic learning and inference in schizophrenia. Schizophr. Res. 127, 115-122.

Bach, D.R., Dolan, R.J., 2012. Knowing how much you don't know: a neural organization of uncertainty estimates. Nat. Rev. Neurosci. 13, 572-586.

Bentall, R.P., Rowse, G., Shryane, N., Kinderman, P., Howard, R., Blackwood, N., Moore, R. Corcoran, R, 2009. The cognitive and affective structure of paranoid delusions: a transdiagnostic investigation of patients with schizophrenia spectrum disorders and depression. Arch. Gen. Psychiatry 66, 236-247.

Blackwood, N., Fytche, D., Simmons, A., Bentall, R., Murray, R., Howard, R., 2004. The cerebellum and decision making under uncertainty. Cogn. Brain Res. 20, 46-53.

Broome, M.R., JOHNS, L.C.D., Valli, I.M.D., Wooley, J.B.M., Tabraham, P.D., Brett, C. Valmaggia, L., Peteres, E., Garety, P.A., McGUIRE, P.K.P., 2007. Delusion formation and reasoning biases in those at clinical high risk for psychosis. Br. J. Psychiatry 191, s38-s42. 
Buck, K.D., Warman, D.M., Huddy, V., Lysaker, P.H., 2012. The relationship of metacognition with jumping to conclusions among persons with schizophrenia spectrum disorders. Psychopathology 45, 271-275.

Deco, G., Rolls, E.T., Albantakis, L., Romo, R., 2013. Brain mechanisms for perceptual and reward-related decision-making. Prog. Neurobiol. http://dx.doi.org/10.1016/j. pneurobio.2012.01.010.

Dimaggio, G., Nicolò, G., Semerari, A., Carcione, A., 2013. Investigating the personality disorder psychotherapy process: the roles of symptoms, quality of affects, emotional dysregulation, interpersonal processes, and mentalizing. Psychother. Res. 23, 624-632

Dura-Bernal, S., Wennekers, T., Denham, S.L., 2012. Top-down feedback in an HMAX-like cortical model of object perception based on hierarchical Bayesian networks and belief propagation. PLoS ONE 7, e48216.

Duvernoy, H.M., 1995. The Human Brainstem and Cerebellum. Surface, Structure, Vascularization and Three Dimensional Sectional Anatomy with MRI. Springer-Verlag, Wien, New York (Ref Type: Serial (Book,Monograph)).

Esslinger, C., Braun, U., Schirmbeck, F., Santos, A., Meyer-Lindenberg, A., Zink, M., Kirsch, P., 2013. Activation of midbrain and ventral striatal regions implicates salience processing during a modified beads task. Plos ONE 8, e58536. http://dx.doi.org/10.1371/journal. pone.0058536.

Fine, C., Gardner, M., Craigie, J., Gold, I., 2007. Hopping, skipping or jumping to conclusions? Clarifying the role of the JTC bias in delusions. Cogn. Neuropsychiatry $12,46-77$.

Fiorillo, C.D., Tobler, P.N., Schultz, W., 2003. Discrete coding of reward probability and uncertainty by dopamine neurons. Science 299, 1898-1902.

Fletcher, P.C., Frith, C.D., 2009. Perceiving is believing: a Bayesian approach to explaining the positive symptoms of schizophrenia. Nat. Rev. Neurosci. 10, 48-58.

Friston, K., 2010. The free-energy principle: a unified brain theory? Nat. Rev. Neurosci. 11, $127-138$

Furl, N., Averbeck, B.B., 2011. Parietal cortex and insula relate to evidence seeking relevant to reward-related decisions. J. Neurosci. 31, 17572-17582.

Garety, P.A., Gittins, M., Jolley, S., Bebbington, P., Dunn, G., Kuipers, E., Fowler, D., Freeman, D., 2012. Differences in cognitive and emotional processes between persecutory and grandiose delusions. Schizophr. Bull. http://dx.doi.org/10.1093/schbul/sbs059.

Gold, J.M., Waltz, J.A., Matveeva, T.M., Kasanova, Z Strauss, G.P., Herbener, E.S., Collins, A.G. Frank, M.J., 2012. Negative symptoms and the failure to represent the expected reward value of actions: Behavioral and computational modeling evidence. Arch. Gen. Psychiatry 69, 129-138.

Goto, Y., Otani, S., Grace, A.A., 2007. The Yin and Yang of dopamine release: a new perspective. Neuropharmacology 53, 583-587.

Grinband, J., Hirsch, J., Ferrera, V.P., 2006. A neural representation of categorization uncertainty in the human brain. Neuron $49,757-763$.

Hemsley, D.R., Garety, P.A., 1986. The formation of maintenance of delusions: a Bayesian analysis. Br. J. Psychiatry 149, 51-56.

Juckel, G., Schlagenhauf, F., Koslowski, M., Filonov, D., Wustenberg, T., Villringer, A., Knutson, B., Kienast, T., Gallinat, J., Wrase, J., Heinz, A., 2006a. Dysfunction of ventral striatal reward prediction in schizophrenic patients treated with typical, not atypical, neuroleptics. Psychopharmacology 187, 222-228.

Juckel, G., Schlagenhauf, F., Koslowski, M., Wustenberg, T., Villringer, A., Knutson, B., Wrase, J., Heinz, A., 2006b. Dysfunction of ventral striatal reward prediction in schizophrenia. Neuroimage 29, 409-416.

Koch, K., Wagner, G., Schachtzabel, C., Schultz, C.C., Guellmar, D., Reichenbach, J.R., Sauer, H., Schloesser, R.G.M., 2011. Neural activation and radial diffusivity in schizophrenia: combined fMRI and diffusion tensor imaging study. Br. J. Psychiatry 198, 223-229.

Krug, A., Cabanis, M., Pyka, M., Pauly, K., Kellermann, T., Walter, H., Wagner, M., Landsberg, M., Shah, N.J., Winterer, G., Wölwer, W., Brinkmeyer, J., Müller, B.W., Kärgel, C., Wiedemann, G., Herrlich, J., Vogeley, K., Schilbach, L., Rapp, A., Klingberg, S., Kircher, T., 2014. Attenuated prefrontal activation during decision-making under uncertainty in schizophrenia: a multi-center fMRI study. Schizophr. Res. 152, 176-183.

Ladegaard, N., Larsen, E.R., Videbech, P., Lysaker, P.H., 2014. Higher-order social cognition in first-episode major depression. Psychiatry Res. http://dx.doi.org/ 10.1016/j.psychres.2013.12.010.

Lee, T.S., Mumford, D., 2003. Hierarchical Bayesian inference in the visual cortex. J. Opt. Soc. Am. A 20, 1434-1448.

Lincoln, T.M., Ziegler, M., Mehl, S., Rief, W., 2010a. The jumping to conclusions bias in delusions: specificity and changeability. J. Abnorm. Psychol. 119, 40-49.

Lincoln, T.M., Lange, J., Burau, J., Exner, C., Moritz, S., 2010b. The effect of state anxiety on paranoid ideation and jumping to conclusions, an experimental investigation. Schizophr. Bull. 36, 1140-1148.

Lunt, L., Bramham, J., Morris, R.G., Bullock, P.R., Selway, R.P., Xenitidis, K., David, A.S., 2012. Prefrontal cortex dysfunction and 'jumping to conclusions': bias or deficit? J. Neuropsychol. 6, 65-78.

Lysaker, P.H.P., Warman, D.M.P., Dimaggio, G.M., Procacci, M.M., LaRocco, V.A.M. Clark, L.K.M., Dike, C.A.M., Nicolo, G.M., 2008. Metacognition in schizophrenia: associations with multiple assessments of executive function. J. Nerv. Ment. Dis. 196, 384-389.

Lysaker, P.H., Erickson, M., Ringer, J., Buck, K.D., Semerari, A., Carcione, A., Dimaggio, G. 2011. Metacognition in schizophrenia: the relationship of mastery to coping insight, self-esteem, social anxiety, and various facets of neurocognition. Br. J. Clin. Psychol. $50,412-424$

Lysaker, P.H., Gumley, A., Luedtke, B., Buck, K.D., Ringer, J.M., Olesek, K., Kukla, M., Leonhardt, B.L., Popolo, R., Dimaggio, G., 2013a. Social cognition and metacognition in schizophrenia: evidence of their independence and linkage with outcomes. Acta Psychiatr. Scand. 127, 239-247.

Lysaker, P.H., Vohs, J., Hillis, J.D., Kukla, M., Popolo, R., Salvatore, G., Dimaggio, G., 2013b. Poor insight into schizophrenia: contributing factors, consequences and emerging treatment approaches. Expert. Rev. Neurother. 13, 785-793.
Moritz, S., Woodward, T.S., 2007. Metacognitive training in schizophrenia: from basic research to knowledge translation and intervention. Curr. Opin. Psychiatr. 20, 619-625. Moritz, S., Van Quaquebeke, N., Lincoln, T.M., 2012. Jumping to conclusions is associate with paranoia but not general suspiciousness: a comparison of two versions of the probabilistic reasoning paradigm. Schizophr. Res. Treat. http://dx.doi.org/10.1155/ 2012/384039.

Morris, R.W., Vercammen, A., Lenroot, R., Moore, L., Langton, J.M., Short, B., Kulkarni, J., Curtis, J., O'Donnell, M., Weickert, C.S., Weickert, T.W., 2012. Disambiguating ventral striatum fMRI-related bold signal during reward prediction in schizophrenia. Mol. Psychiatry 17, 280-289.

Murray, G.K., 2011. The emerging biology of delusions. Psychol. Med. 41, 7-13.

Murray, G.K., Corlett, P.R., Clark, L., Pessiglione, M., Blackwell, A.D., Honey, G., Jones, P.B Bullmore, E.T., Robbins, T.W., Fletcher, P.C., 2008. Substantia nigra/ventral tegmental reward prediction error disruption in psychosis. Mol. Psychiatry 13, 239-276.

Ormrod, J., Shaftoe, D., Cavanagh, K., Freeston, M., Turkington, D., Price, J., Dudley, R., 2011. A pilot study exploring the contribution of working memory to "jumping to conclusions" in people with first episode psychosis. Cogn. Neuropsychiatry 17, 97-114.

Peters, E., Garety, P., 2006. Cognitive functioning in delusions: a longitudinal analysis. Behav. Res. Ther. 44, 481-514.

Rubio, J.L., Ruiz-Veguilla, M., Hernandez, L., Barrigon, M.L., Salcedo, M.D., Moreno, J.M Gomez, E., Moritz, S., Ferrin, M., 2011. Jumping to conclusions in psychosis: a faulty appraisal. Schizophr. Res. 133, 199-204.

Schultz, W., 2013. Updating dopamine reward signals. Curr. Opin. Neurobiol. 23, 229-238.

Schultz, W., Preuschoff, K. Camerer, C. Hsu, M. Fiorillo, C.D., Tobler, P.N., Bossaerts, P. 2008. Explicit neural signals reflecting reward uncertainty. Phil. Trans. R. Soc. London 363, 3801-3811.

So, S.H., Garety, P.A., Peters, E.R., Kapur, S., 2010. Do antipsychotics improve reasoning biases? A review. Psychosom. Med. 72, 681-693.

So, S.H., Freeman, D. Dunn, G., Kapur, S., Kuipers, E., Bebbington, P., Fowler, D., Garety, P.A., 2012. Jumping to conclusions, a lack of belief flexibility and delusional conviction in psychosis: a longitudinal investigation of the structure, frequency, and relatedness of reasoning biases. J. Abnorm. Psychol. 121, 129-139.

So, S.H., Peters, E.R., Swendson, J., Garety, P.A., Kapur, S., 2014 Mar 30. Changes in delusions in the early phase of antipsychotic treatment - an experience sampling study. Psychiatry Res. 215 (3), 568-573. http://dx.doi.org/10.1016/j.psychres.2013. 12.033 Epub 2013 Dec 24

Speechley, W.J., Whitman, J.C., Woodward, T.S., 2010. The contribution of hypersalience to the "jumping to conclusions" bias associated with delusions in schizophrenia. J. Psychiatry Neurosci. 35, 7-17.

Tas, C., Brown, E.C., Esen-Denaci, A., Lysaker, P.H., Bruene, M., 2012. Intrinsic motivation and metacognition as predictors of learning potential in patients with remitted schizophrenia. J. Psychiatr. Res. 46, 1086-1092.

Tas, C., Brown, E., Cubukcuoglu, Z., Aydemir, O., Danaci, A.E., Brüne, M., 2013. Towards an integrative approach to understanding quality of life in schizophrenia: the role of neurocognition, social cognition, and psychopathology. Compr. Psychiatry 54, 262-268.

Tobler, P.N., Fiorillo, C.D., Schultz, W., 2005. Adaptive coding of reward value by dopamine neurons. Science 307, 1642-1645.

Van Donkersgoed, R.J., De Jong, S., van der Gaag M.,Aleman, A. Lysaker, P.H., Wunderink L., Pijnenborg, G., 2014. A manual-based individual therapy to improve metacognition in schizophrenia: protocol of a multi-center RCT. BMC Psychiatry 14, 27.

Veckenstedt, R., Randjbar, S., Vitzthum, F., Hottenrott, B., Woodward, T.S., Moritz, S., 2011. Incorrigibility, jumping to conclusions, and decision threshold in schizophrenia. Cogn. Neuropsychiatry 16, 174-192.

Visscher, K.M., Miezin, F.M., Kelly, J.E., Buckner, R.L., Donaldson, D.I., McAvoy, M.P., Bhalodia, V.M., Petersen, S.E., 2003. Mixed blocked/event-related designs separate transient and sustained activity in fMRI. Neuroimage 19, 1694-1708.

Vohs, J.L., Lysaker, P.H., Francis, M.M., Hamm, J., Buck, K.D., Olesek, K., Outcalt, J., Dimaggio, G., Leonhardt, B., Liffick, E., Mehdiyoun, N., Breier, A., 2014. Metacognition, social cognition, and symptoms in patients with first episode and prolonged psychoses. Schizophr. Res 153, 54-59.

Walter, H., Blecker, C., Kirsch, P., Sammer, G., Schienle, A., Startk, R., Vaitl, D., 2003. MARINA: an easy to use tool for the creation of MAsks for Region of INterest Analyses [abstract]. Presented at the 9th International Conference on Functional Mapping of the Human Brain, June 19-22, 2003, New York, NY. Available on CD-Rom. Neuroimage 19 (Congress abstract)

Waltz, J.A., Frank, M.J., Robinson, B.M., Gold, J.M., 2007. Selective reinforcement learning deficits in schizophrenia support predictions from computational models of striatalcortical dysfunction. Biol. Psychiatry 62, 756-764

Weickert, T.W., Goldberg, T.E., Callicott, J.H., Chen, Q., Apud, J.A., Das, S., Zoltick, B.J., Egan, M.F., Meeter, M., Myers, C., Gluck, M.A., Weinberger, D.R., Mattay, V.S., 2009. Neural correlates of probabilistic category learning in patients with schizophrenia. J. Neurosci. 29, 1244-1254.

Woodward, T.S., Mizrahi, R., Menon, M., Christensen, B.K., 2009a. Correspondences between theory of mind, jumping to conclusions, neuropsychological measures and the symptoms of schizophrenia. Psychiatry Res. 170, 119-123.

Woodward, T.S., Munz, M., LeClerc, C., Lecomte, T., 2009b. Change in delusions is associated with change in "jumping to conclusions". Psychiatry Res. 170, 124-127.

Xi, C., Zhu, Y., Niu, C., Zhu, C., Lee, T.M., Tian, Y., Wang, K., 2011. Contributions of subregions of the prefrontal cortex to the theory of mind and decision making. Behav. Brain Res. 221, 587-593.

Yu, A.J., Dayan, P., 2005. Uncertainty, neuromodulation, and attention. Neuron 46, 681-692.

Ziauddeen, H., Murray, G.K., 2010. The relevance of reward pathways for schizophrenia. Curr. Opin. Psychiatr. 23, 91-96.

Ziegler, M., Rief, W., Lincoln, T.M., 2012. Does "jumping to conclusions" contirbute to the development and maintenance of delusions? A systematic and quantitative review. Z Psychiatr. Psychol. Psychother. 57, 125-136. 